\title{
Peritonitis due to Mycobacterium abscessus in peritoneal dialysis patients: case presentation and mini-review
}

\author{
Erina Ono ${ }^{1}$, Eiichiro Uchino ${ }^{1}$, Keita P. Mori ${ }^{1}{ }^{2 *}$, Hideki Yokoi ${ }^{1}$, Naohiro Toda ${ }^{1}$, Kenichi Koga ${ }^{3}$, Masato Kasahara ${ }^{4}$, \\ Takeshi Matsubara ${ }^{1}$ and Motoko Yanagita, ${ }^{1,2}$
}

\begin{abstract}
Background: Peritoneal dialysis (PD)-associated peritonitis caused by nontuberculous mycobacteria (NTM), including Mycobacterium abscessus (M. abscessus), is a rare but serious complication that forces PD to be withdrawn. Several cases of peritonitis by NTM have been reported, and optimal treatment has not yet been established.

Case presentations: We report two cases of PD-associated peritonitis caused by M. abscessus. In both cases, peritonitis developed after an exit-site infection. The patients did not have any typical signs of peritonitis or an elevated nucleated cell count of the dialysis effluent in the early phase. In addition, effluent cultures were negative at admission in both cases, although M. abscessus was identified in effluent cultures in the late phase. One patient recovered after the PD catheter was removed, and multi-antibiotic treatment was administered for 6 months. The other patient subsequently developed encapsulating peritoneal sclerosis (EPS) 16 months after the onset of infection. In addition, the EPS was complicated by intestinal perforation into infected ascites. The infection resolved with antibiotic treatment and octreotide administration to diminish bowel leakage into the infected cavity.

Conclusions: The combination of amikacin, clarithromycin, and imipenem/cilastatin with PD catheter removal may be effective for the treatment of $M$. abscessus PD-associated peritonitis. The prognosis of $M$. abscessus-induced peritonitis is generally poor, and it is of note that residual encapsulated ascites in the peritoneal cavity after treatment may increase the risk of infection recurrence or EPS development.
\end{abstract}

Keywords: Encapsulating peritoneal sclerosis, Exit-site infection, Mycobacterium abscessus, Nontuberculous mycobacteria, Peritoneal dialysis, Peritonitis

\section{Background}

Peritoneal dialysis (PD) is a standard modality of renal replacement therapy for end-stage renal disease (ESRD). Clinicians, however, often encounter cases where PD is forced to be withdrawn as a result of factors such as peritonitis and volume overload. PD-associated peritonitis is one of the most significant complications caused by the contamination of dialysate and spread of exit-site infection (ESI) or tunnel infection. Although PD-associated peritonitis is typically caused by bacteria such as coagulase-

\footnotetext{
* Correspondence: moryk@kuhp.kyoto-u.ac.jp

'Department of Nephrology, Graduate School of Medicine, Kyoto University,

54 Shogoin Kawahara-cho, Sakyo-ku, Kyoto 606-8507, Japan

${ }^{2}$ Medical Innovation Center, Graduate School of Medicine, Kyoto University,

53 Shogoin Kawahara-cho, Sakyo-ku, Kyoto 606-8507, Japan

Full list of author information is available at the end of the article
}

negative staphylococcus and Staphylococcus aureus, which can be treated with antibiotics, an infection caused by acid-fast bacterial or fungal peritonitis requires PD catheter removal in addition to the administration of multiple antibacterial drugs or antifungal drugs, respectively. Importantly, the number of cases of peritonitis caused by nontuberculous mycobacteria (NTM), which are acid-fast bacilli, is increasing [1]. Mycobacterium abscessus (M. abscessus) is a species of rapidly growing NTM that exists in environments such as dirty water, soil, food, and animals [2]. Although $M$. abscessus is known to cause pulmonary, skin, and soft tissue infections, peritonitis caused by $M$. abscessus is reported to be rare [2, 3]. A recent report showed that antibiotics abuse could result in the spread of M. abscessus infections [4]. Despite the rapid growth of $M$.

(C) The Author(s). 2018 Open Access This article is distributed under the terms of the Creative Commons Attribution 4.0 International License (http://creativecommons.org/licenses/by/4.0/), which permits unrestricted use, distribution, and 
abscessus, it is difficult to diagnose and treat, especially at onset. Herein, we report two cases of peritonitis caused by M. abscessus and also review M. abscessus-associated peritonitis in the literature.

\section{Case presentations}

\section{Case 1}

A 59-year-old female had been receiving PD treatment for 1 year because of ESRD due to nephrosclerosis. She had no history of diabetes mellitus, immunodeficiency, mycobacterial infection, or peritonitis. She had not been treated with immunosuppressants or anticancer drugs. Peritoneal equilibrium test (PET) showed high average transporter status 1 year before admission. She had been diagnosed with ESI and tunnel infection 2 months before admission. The gram stain and culture of the exit site for general bacteria were negative; acid-fast bacilli (AFB) stain was not performed. She was treated with surgical drainage, topical gentamicin, and systemic antibiotics by intravenous injection. Because she was unresponsive to these treatments and the results of echographic examination indicated that the infection was affecting the area from the exit site to the external cuff, but not to the catheter tunnel between the internal and external cuff, we performed exit-site translocation. No improvement in the skin infection was observed, however. She was admitted to Kyoto University Hospital for further treatment.

On admission, her body temperature was $36.4^{\circ} \mathrm{C}$, blood pressure was $116 / 86 \mathrm{mmHg}$, and heart rate was 89 beats/ min. Her urine volume was $700 \mathrm{~mL} /$ day, but the creatinine clearance was not measured. She had red, painful, and swollen wounds on the skin over the sites of the internal cuff, the external cuff, and the previously used external cuff, with purulent discharge and ulceration (Fig. 1 A). There were no physical signs of peritonitis. Laboratory data are summarized in Table 1 . Her dialysis effluent was normal in appearance, and her gram stain was negative (Table 1). Blood, sputum, and stool cultures were all negative. Computed tomography (CT) showed no focal signs of inflammation, except for increased density of subcutaneous fat around the PD catheter. She was diagnosed with refractory ESI and tunnel infection. As her skin infection was not improved by the combination of intravenous cefazolin and ceftazidime, replacement of the PD catheter with debridement of the subcutaneous tissues around the catheter was performed on the 5th day of admission. She developed fever and chills on the 11th day of admission, however, and diarrhea, nausea, and vomiting on the 14th day of admission. As methicillin-resistant Staphylococcus aureus (MRSA) or Pseudomonas aeruginosa infection was suspected, we began intravenous cefepime and teicoplanin.
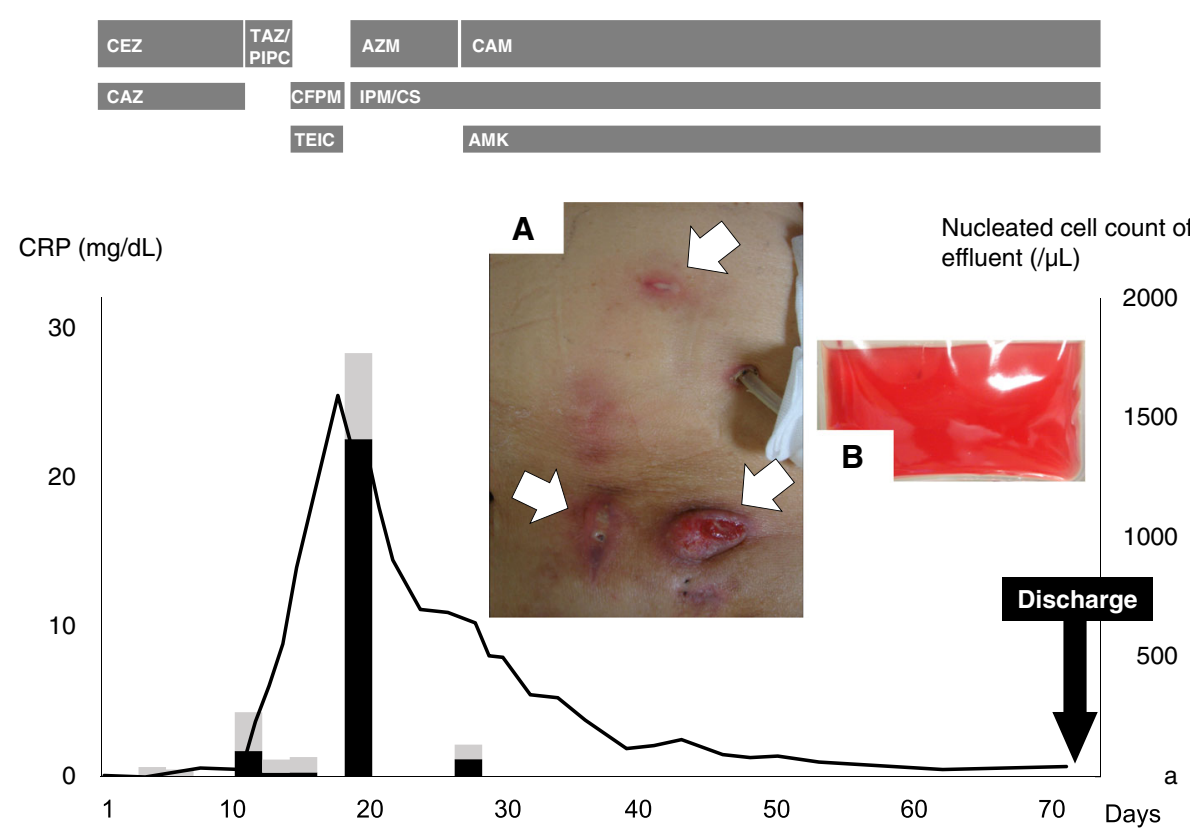

Fig. 1 The clinical course of case 1. Horizontal axis indicates hospitalization. Bar graph indicates effluent nucleated cell count (black and gray bars represent ratios of neutrophils other than white blood cells, respectively). Line graph indicates the trend in serum C-reactive protein level. On the 1st day of admission, the patient had red, painful, and swollen skin over the sites of the internal cuff, the external cuff, and the previously used external cuff, with purulent discharge and ulceration (a). The dialysis effluent on the 25th day of admission was cloudy and bloody (b). The PD catheter was removed on the 29th day of admission. The patient was discharged on the 77th day of admission. AMK, amikacin; AZM, azithromycin; CAM, clarithromycin; CAZ, ceftazidime; CEZ, cefazolin; CFPM, cefepime; CRP, C-reactive protein; IPM/CS, imipenem/cilastatin; PD, peritoneal dialysis; TAZ/PIPC, tazobactam/piperacillin; TEIC, teicoplanin 
Table 1 Laboratory data of case 1 and case 2

\begin{tabular}{|c|c|c|c|}
\hline & Case 1 & Case 2 & Reference range \\
\hline \multicolumn{4}{|l|}{ Blood } \\
\hline White blood cell count $(/ \mu \mathrm{L})$ & 3600 & 11,970 & 2900-7800 \\
\hline Neutrophil (\%) & 64.9 & 95.3 & $46-62$ \\
\hline Hemoglobin (g/dL) & 9.4 & 11.2 & $10.1-14.7$ \\
\hline Platelet count $\left(\times 10^{3} / \mu \mathrm{L}\right)$ & 221 & 179 & $131-384$ \\
\hline Albumin (g/dL) & 3.2 & 1.7 & $3.9-5.1$ \\
\hline Creatinine (mg/dL) & 6.4 & 9.19 & $0.4-0.8$ \\
\hline Blood urea nitrogen (mg/dL) & 47 & 48 & $8-22$ \\
\hline Glucose (mg/dL) & 115 & 137 & $78-110$ \\
\hline Sodium (mEq/L) & 136 & 129 & $136-144$ \\
\hline Potassium (mEq/L) & 3.7 & 2.9 & $3.6-4.8$ \\
\hline Chloride (mEq/L) & 100 & 89 & 99-109 \\
\hline Calcium (mg/dL) & 8.8 & 7.9 & $8.5-9.9$ \\
\hline Phosphorus (mg/dL) & 5.3 & 3.8 & $2.6-4.5$ \\
\hline C-reactive protein (mg/dL) & 0.1 & 26.6 & $\leqq 0.2$ \\
\hline$\beta$-D-Glucan (pg/mL) & $<3$ & $<3$ & $<11$ \\
\hline \multicolumn{4}{|l|}{ Dialysis effluent } \\
\hline Nucleated cell count $(/ \mu \mathrm{L})$ & 40 & 160 & \\
\hline Neutrophil (\%) & 2 & 87 & \\
\hline Gram stain & Negative & Negative & Negative \\
\hline Admission day on which acid-fast bacilli stain was positive & Day 18 & Day 10 & \\
\hline
\end{tabular}

On the 18th day of admission, the effluent became cloudy with fibrin precipitation and AFB was positive. The culture of the internal cuff collected on the 1st day of admission was positive for $M$. abscessus. Her antibiotics were changed to oral azithromycin and intravenous imipenem/cilastatin based on the results of antimicrobial susceptibility testing. Her renal replacement therapy was changed from PD to hemodialysis (HD). Although the inflammatory reaction was improving, CT scan detected enhancement of the peritoneum and an increased density of the mesentery. These findings indicated the formation of adherent biofilm by bacteria in the second PD catheter. Although we suspected encapsulating peritoneal sclerosis (EPS) because her effluent was bloody and encapsulated ascites were detected by $\mathrm{CT}$, we decided to remove the catheter to fully resolve the peritonitis on the 29th day of admission (Fig. 1 B). After removal, her symptoms and inflammatory reaction improved. Although encapsulated ascites remained on the 44th day of admission (Fig. 3 A), they finally disappeared after treatment. She was discharged on the 77th day of admission with daily oral clarithromycin and intermittent intravenous amikacin and imipenem/ cilastatin at each HD session (Fig. 1). After a total of 6 months of antibiotics administration, she has had no recurrence of peritonitis or ileus and continues to undergo maintenance HD.

\section{Case 2}

A 67-year-old male had been on PD for 5 years because of ESRD due to nephrosclerosis. He had no history of diabetes mellitus, immunodeficiency, mycobacterial infection, or peritonitis. He had not been treated with immunosuppressants or anticancer drugs. PET showed high average transporter status 6 months before admission. He had been diagnosed with ESI 6 months before admission due to purulent discharge at the exit site. Gram stain and culture of the exit site for general bacteria were negative, but AFB stain was not performed. His ESI improved with topical gentamicin. He had general malaise, anorexia, and cough beginning 2 months before admission, however, and was nearly bedridden for the month prior to admission due to his worsening symptoms. He was admitted to Kyoto University Hospital for diarrhea, a $3.2-\mathrm{kg}$ loss of body weight (previous mean body weight was $70 \mathrm{~kg}$ ), and mild abdominal pain for 3 days.

On admission, his body temperature was $37.4{ }^{\circ} \mathrm{C}$, blood pressure was $114 / 59 \mathrm{mmHg}$, and heart rate was 77 beats $/ \mathrm{min}$. His body weight was $5 \mathrm{~kg}$ less than that before the illness began. He was also anuric. Physical examination did not reveal any remarkable signs except for redness and purulent discharge at the exit site (Fig. 2 A). Laboratory data are shown in Table 1 . Fibrin precipitation was present in the effluent, but it was not bloody 


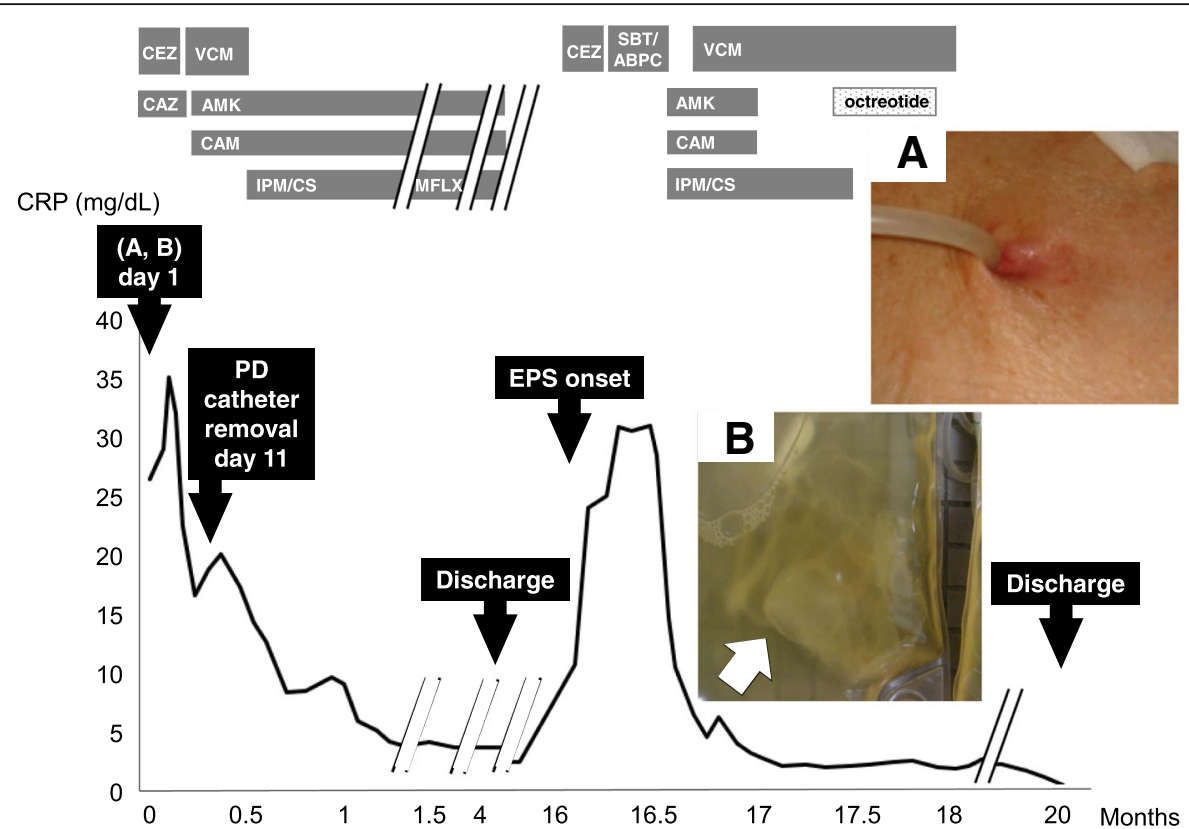

Fig. 2 The clinical course of case 2. Horizontal axis indicates hospitalization. Line graph indicates the trend in serum C-reactive protein level. On the 1st day of admission, the exit site was red with purulent discharge (a). The dialysis effluent on the 1st day of admission had fibrin precipitation without blood (b). The PD catheter was removed on the 11th day of admission. The patient was discharged on the 135th day of admission. The patient developed encapsulating peritoneal sclerosis 16 months after the onset of peritonitis, and he was discharged 20 months after the onset of peritonitis. AMK, amikacin; CAM, clarithromycin; CAZ, ceftazidime; CEZ, cefazolin; CRP, C-reactive protein; EPS, encapsulating peritoneal sclerosis; IPM/CS, imipenem/cilastatin; MFLX, moxifloxacin; SBT/ABPC, sulbactam/ampicillin; VCM, vancomycin

(Fig. 2 B). The effluent nucleated cell count was 160 cells/ $\mu \mathrm{L}$ (87\% neutrophils), and a gram stain was negative (Table 1). Blood, sputum, and stool cultures were all negative. On CT, he showed no focal signs of inflammation except for increased density of the subcutaneous fat around the PD catheter that extended from the exit site to the entrance into the peritoneal cavity, indicating a tunnel infection.

He was diagnosed with peritonitis based on the abnormal effluent and tunnel infection detected by CT. He was started on intravenous cefazolin and ceftazidime empirically. Although cefazolin was changed to intravenous vancomycin due to ineffectiveness, he had no signs of improvement on the 4th day of admission. The nucleated cell count of the effluent increased to $480 / \mu \mathrm{L}(62 \%$ neutrophils), and the effluent culture was negative for fungus and MRSA. Therefore, on the 5th day of admission, intravenous amikacin and oral clarithromycin were started, ceftazidime was discontinued, and PD was changed to HD because of a suspected atypical infection. On the 6th day of admission, he showed signs of ileus and was treated with insertion of a nasogastric tube. CT showed focal encapsulated ascites in the peritoneal cavity and possible EPS. On the 10th day of admission, culture effluent taken on the 4th day of admission was found to be AFB-stain positive and was identified as $M$. abscessus on the 17th day of admission. On the 11th day of admission, the PD catheter was removed (culture was positive for $M$. abscessus), and vancomycin was changed to daily intravenous imipenem/cilastatin according to antimicrobial susceptibility testing on the 17th day of admission. After starting imipenem/cilastatin, his condition gradually improved (Fig. 2). Oral food intake was recommended on the 23rd day of admission. His blood test results and inflammatory reaction improved although the encapsulated ascites remained on the 40th day of admission (Fig. 3 B). Daily intravenous imipenem/ cilastatin was changed to daily oral moxifloxacin on the 59th day of admission in preparation for ambulatory care. On the 135th day of admission, he was discharged. Daily oral clarithromycin and moxifloxacin and intermittent intravenous amikacin at each HD session were continued for a total of 6 months, but he developed a fever and abdominal pain 16 months after the onset of peritonitis. We performed an abdominal CT and made a diagnosis of ileus due to the enlarged encapsulated ascites and dilated intestinal tracts with fluid levels (Fig. 4 A-C). The culture of the ascites was positive for Escherichia coli, Enterococcus faecium, and Enterococcus avium, but not for $M$. abscessus. We speculated that multiple intestinal bacteria had inflowed into the encapsulated ascites by intestinal perforation due to EPS, resulting in microbial substitution. After he was treated by the drainage of the encapsulated ascites, stopping oral food 

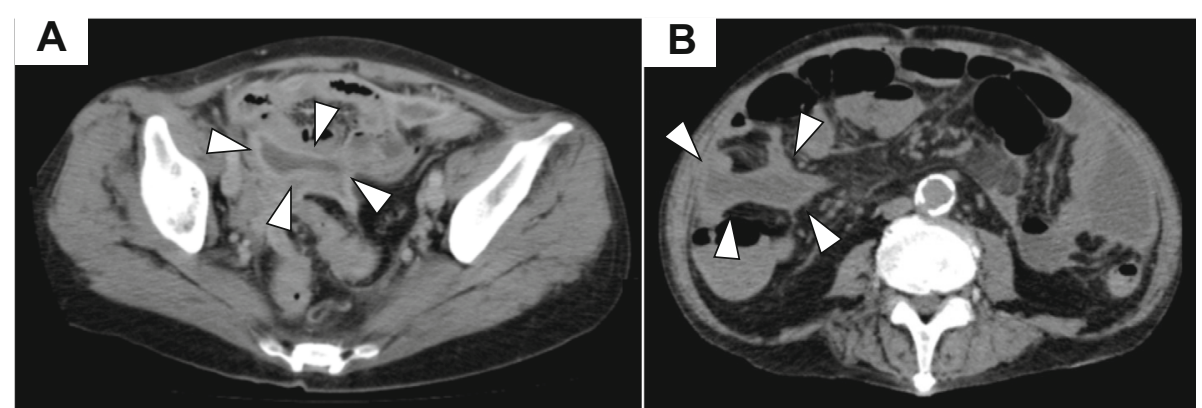

Fig. 3 Encapsulated ascites detected by computed tomography in both cases. On the 48th day of admission in case 1 with C-reactive protein (CRP) $1.3 \mathrm{mg} / \mathrm{dL}$ (a). On the 40th day of admission in case 2 with CRP $3.6 \mathrm{mg} / \mathrm{dL}$ (b). White arrowheads indicate encapsulated ascites

intake, and intravenous antibiotics use, the inflammatory reaction improved and the size of the ascites was reduced. Leaking oral contrast media into the retained ascites revealed intestinal perforation, however (Fig. 4 D). We believe that the infection of the encapsulated ascites was complicated by intestinal rupture due to EPS. To diminish bowel leakage into the infected cavity, we added a continuous subcutaneous infusion of octreotide $300 \mu \mathrm{g} / 24 \mathrm{~h}$ for treatment. After 3.5 months of antibiotic treatment and octreotide for 3 weeks, the fistula between the encapsulated ascites and bowel closed, and the patient was discharged 4 months later.

\section{Mini-review of PD-associated peritonitis by M. abscessus Etiology}

Peritonitis by $M$. abscessus is a rare complication among PD patients. Including our cases, there are only 18 cases reported in the literature, mostly from Asia, Australia, the Netherlands, and the USA (Table 2) [4-11], as Yang et al. reported previously [5]. NTM infection tends to occur in patients with immunodeficiency and implanted artificial materials. Among patients treated with PD, under-dialysis, poor residual renal function, and a history of broadspectrum antibiotics use have been reported as risk factors $[2,7,12]$. Recently, topical gentamicin use at the exit site
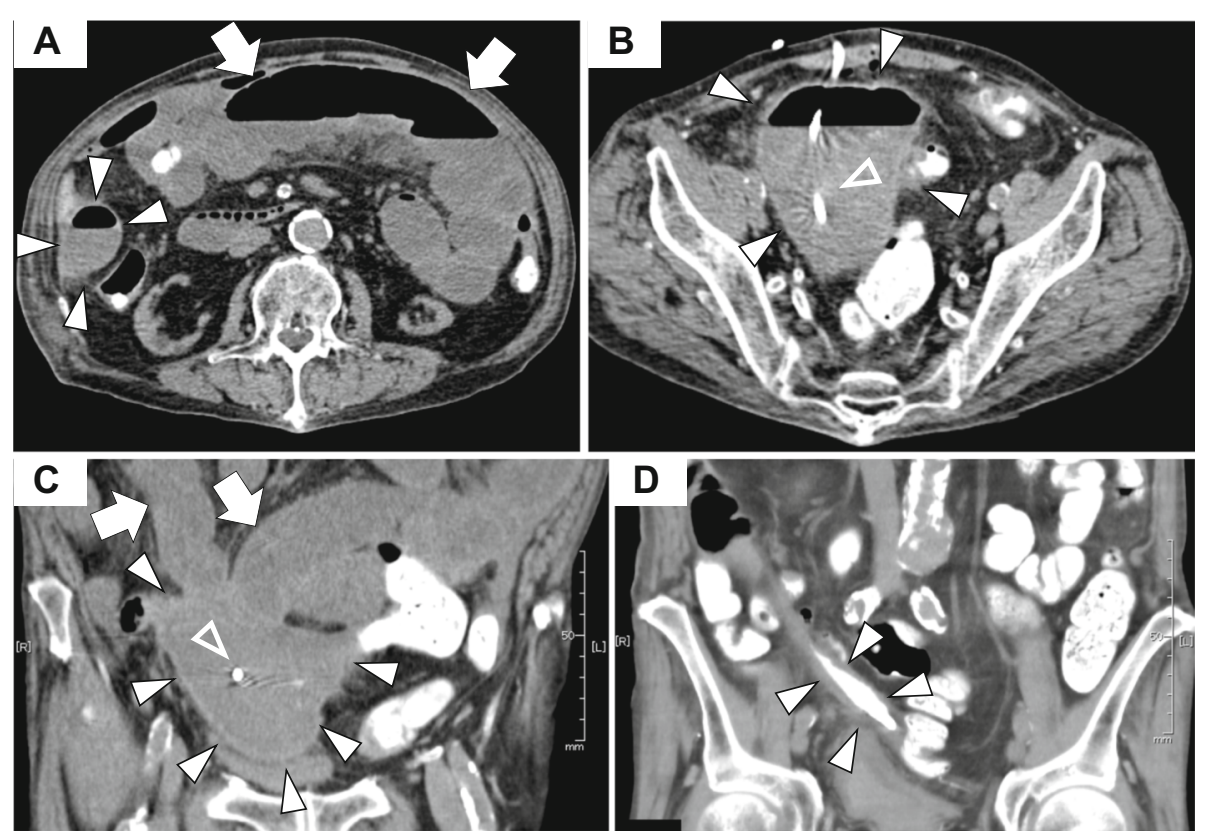

Fig. 4 Abdominal computed tomography images 16 months after the onset of peritonitis in case 2. Dilated intestinal tracts with fluid levels suggest ileus (a). It is of note that encapsulated ascites were observed in the right abdomen. Enlarged encapsulated ascites with a drainage catheter and dilated intestinal tracts in coronal sections (b), in horizontal sections (c). The ascites appear to be adjacent to the dilated intestinal tracts. After drainage, the encapsulated ascites were reduced in size, but oral contrast leaking into the residual encapsulated ascites suggested intestinal perforation (d). White arrows indicate the intestinal tract, white arrowheads indicate the encapsulated ascites, and open white arrowheads indicate the drainage catheter 
$\begin{array}{ll}\text { Ono et al. Renal Replacement Therapy } & \text { (2018) } 4: 52\end{array}$

Page 6 of 10

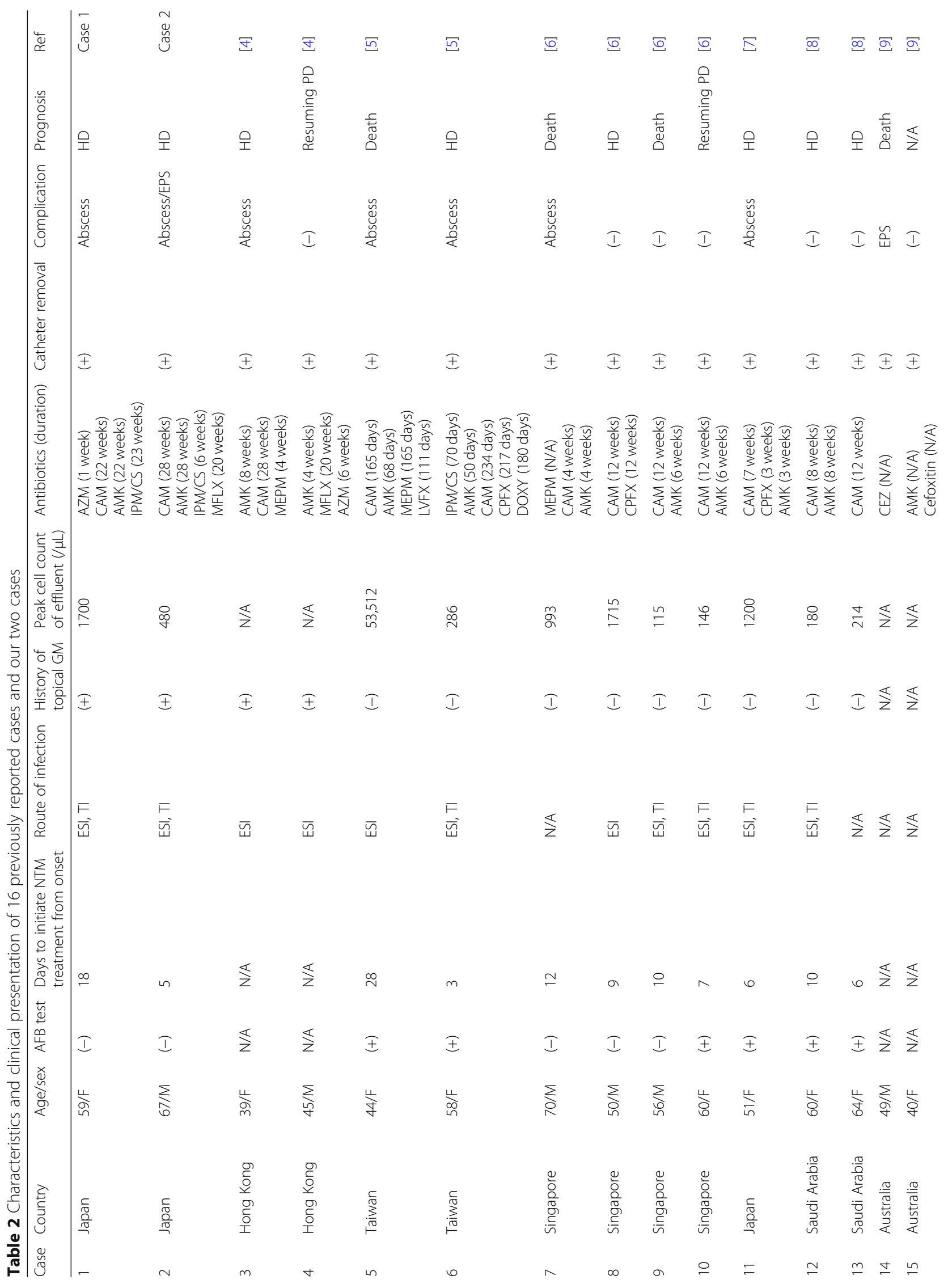


Ono et al. Renal Replacement Therapy

(2018) 4:52

Page 7 of 10

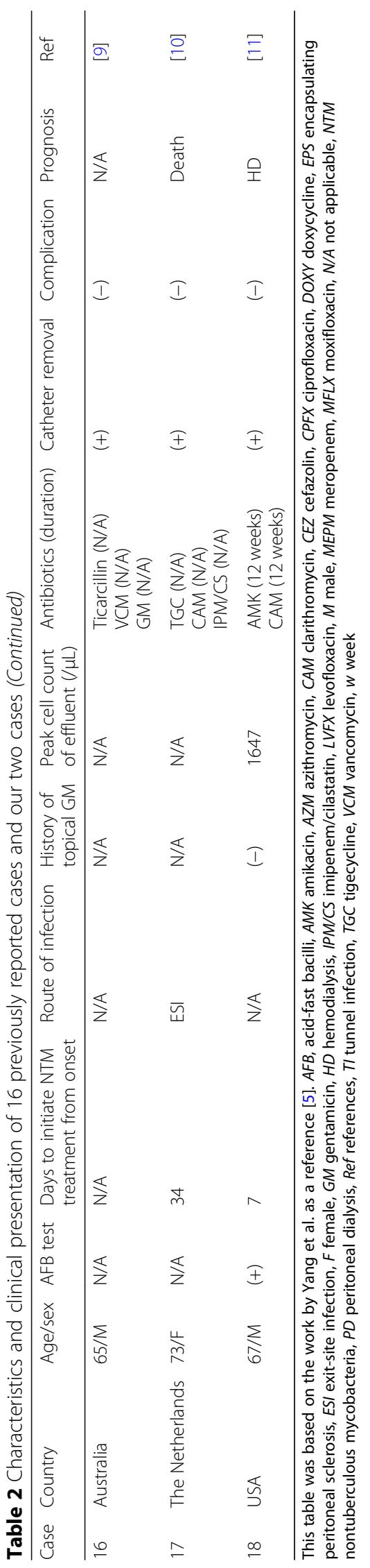


has been reported to increase the risk of NTM infection [4]. M. abscessus lives in environments that include water, soil, and dust, and may cause ESI. M. abscessus proliferating at the exit site might cause peritonitis. The frequency of ESI is high in peritonitis caused by M. abscessus [6]. In previous reports, ESI preceded peritonitis in 10 out of 16 cases (no records in 6 cases), and tunnel infection also preceded peritonitis in 5 cases [4-11]. Therefore, ESI by M. abscessus tends to spread subcutaneously into the peritoneal cavity of patients. Although we could not confirm M. abscessus infection in the exit site in case 2, the patient's ESI was treated by topical gentamicin 6 months before the onset of peritonitis, suggesting that it might increase the risk of $M$. abscessus infection. Although we only found ESI and no obvious signs of tunnel infection on physical examination, CT scan detected tunnel infection in case 2. Hence, we speculate that $M$. abscessus in ESI can spread rapidly into the abdominal cavity via the catheter tunnel before the emergence of typical signs of tunnel infection commonly found by physical examination.

As described above, it has been assumed that $M$. abscessus infection spreads from the environment. A recent report using next-generation genome sequencing, however, revealed that three substrains of $M$. abscessus are almost genetically identical. Moreover, as endemic areas of the substrains were close to each other, the authors concluded that they were clones that had arisen from direct humanto-human transmission [13]. This transmission could occur by infection routes such as cough aerosols by infected patients [13]. Therefore, although there has been no obvious evidence indicating person-to-person transmission, medical staff should be aware of this possibility [14].

\section{Symptoms}

In comparison to typical bacterial peritonitis, NTM peritonitis presents with fewer symptoms, such as abdominal pain. In previous reports of a total of 16 cases, there were 7 cases with abdominal pain, 8 with fever, 3 with diarrhea, 2 with nausea and vomiting, and 1 with cough (5 cases had no records of symptoms) [4-11]. None of our patients had severe abdominal pain, although they had atypical signs such as malaise and cough.

\section{Diagnosis}

According to the International Society for Peritoneal Dialysis Peritonitis Recommendations, peritonitis is generally diagnosed with clinical features consistent with peritonitis during PD treatment, such as elevation of the effluent nucleated cell count (100 cells $/ \mu \mathrm{L}$ with $>50 \%$ neutrophils) and positive effluent culture [1]. Among 16 cases in previous reports and our cases of peritonitis with $M$. abscessus (except for 6 cases without data available), the median of nucleated effluent cell count was 736.5 cells $/ \mu \mathrm{L}$, although in 3 cases, the cell count was only approximately 100 cells/ $\mu \mathrm{L}$ [4-11]. Negative AFB staining in peritonitis caused by NTM is common. Of 12 recorded cases, only 7 had positive AFB staining; the positive predictive value was thus only $58 \%$. The median of the time to initiate specialized treatment for $M$. abscessus from the onset of any symptoms was 9 days, and the longest time was 5 weeks among 13 recorded cases [4-11]. Although M. abscessus is classified as a rapidly growing NTM, it requires at least 4 days to detect this organism by culture, whereas bacteria generally require only a few days [2]. As it took 18 days in case 1 and 10 days in case 2 to prove a mycobacterium infection, AFB staining and culture should be performed at the time of diagnosis of ESI or tunnel infection. Generally, the mycobacterial culture tends to be less sensitive than the culture for common bacteria. Therefore, we should have repeatedly performed mycobacterial cultures during treatment in case 1 because of the culture-negative refractory exit site and tunnel infection. Furthermore, cultures should be performed repeatedly, particularly in atypical cases.

\section{Treatment}

There are no current recommendations for the treatment of peritonitis by $M$. abscessus. According to the American Thoracic Society guidelines of 2007 for pneumonia, $M$. abscessus is sensitive to clarithromycin, amikacin, cefoxitin, linezolid (limited), and imipenem/cilastatin (limited). For severe infection of the skin and soft tissue, the combination therapy of clarithromycin (or azithromycin) and others (amikacin, cefoxitin, or imipenem/cilastatin) for more than 4 months is recommended. For PD, however, removal of the PD catheter and debridement are important and probably essential for recovery [15]. In all 16 previous cases, the PD catheters were removed, and $\geq 2$ antibiotics were used for between 4 and 34 weeks. In addition, clarithromycin, amikacin, and/or imipenem/cilastatin were used in 14 cases [4-11]. In our two cases, the PD catheters were removed and treatment with oral clarithromycin, intravenous imipenem/cilastatin (changed to oral moxifloxacin before discharge in case 2), and intermittent intravenous amikacin was continued for a total of 6 months, resulting in recovery from ESI, tunnel infection, and peritonitis.

\section{Prognosis}

The prognosis of peritonitis caused by M. abscessus has been reported to be poorer than that caused by other NTM infections [5]. Jiang et al. reported a case of sclerosing peritonitis 5 months after diagnosis of PD peritonitis by M. abscessus, although their description lacked details of the clinical course [9]. Ours is the first report to describe the clinical course of EPS occurrence in detail (case 2). Furthermore, the finding that the encapsulated ascites remained after treatment in case 2 seemed noteworthy. Therefore, we emphasized the risk of M. abscessus-induced peritonitis developing into EPS, and the possibility of 
encapsulated ascites as a serious sign for the poor prognosis of peritonitis such as EPS.

\section{Discussion}

We experienced two cases of $M$. abscessus-induced peritonitis with a history of topical gentamicin use at the exit sites. Although there has been no evidence that $M$. abscessus infection can occur at the exit sites as a direct result of topical gentamicin, frequent use appears to cause microbial substitution at the exit sites. It is of note that, in case 2, CT scan detected increased density around the catheter tunnel although we could not identify any typical physical findings indicating skin infection. We speculated that peritonitis could develop via ESI and tunnel infection. $M$. abscessus was reported to be resistant to chlorhexidine and alcohol, which are the most frequently used disinfectants, whereas alcoholic povidone-iodine is effective against it [16, 17]. The frequent use of alcoholic povidone-iodine, however, could cause skin injury, which could increase the risk of ESI caused by $M$. abscessus. Hence, it might be important to avoid the excessive use of antiseptic solution and to keep the exit site clean with careful monitoring instead.

We emphasize the possibility of EPS after peritonitis by $M$. abscessus, particularly with the encapsulated ascites detected by CT during treatment. It is not well known whether peritonitis caused by $M$. abscessus contributes to EPS occurrence or not. Evidence from animal experiments demonstrates that vascular endothelial growth factor (VEGF) and transforming growth factor- $\beta$ (TGF- $\beta$ ) can play crucial roles in the induction of peritoneal fibrosis $[18,19]$. Recently, it was reported that serum VEGF levels were higher in patients with NTM-lung diseases than in patients with NTM-pulmonary colonization alone. [20] Interestingly, another group reported that serum levels of cytokines, including TGF- $\beta$, in patients with $M$. abscessus infection were higher than those in patients without $M$. abscessus infection [21]. Therefore, peritonitis caused by M. abscessus could carry a high risk of EPS occurrence compared to peritonitis caused by non-NTM bacteria or other NTMs.

\section{Conclusions}

Patients with peritonitis caused by M. abscessus do not always have the typical signs observed in common bacterial peritonitis, such as abdominal pain and elevated effluent nucleated cell count. Therefore, AFB staining and culture for NTM should be performed at presentation and repeated until peritonitis by NTM is excluded. The long-term use of topical gentamicin may be a possible risk factor for M. abscessus infection. After diagnosing peritonitis by $M$. abscessus, empiric treatment with multiple antibiotics containing clarithromycin, amikacin, and imipenem/cilastatin coupled with the immediate removal of the PD catheter is strongly recommended. As there is an increased risk of EPS after M. abscessus-induced peritonitis, long-term use of antibiotics and careful observation are needed, particularly in cases with remaining encapsulated ascites after treatment.

\section{Abbreviations}

AFB: Acid-fast bacilli; AMK: Amikacin; AZM: Azithromycin;

CAM: Clarithromycin; CAZ: Ceftazidime; CEZ: Cefazolin; CFPM: Cefepime;

CPFX: Ciprofloxacin; CRP: C-reactive protein; CT: Computed tomography; DOXY: Doxycycline; EPS: Encapsulating peritoneal sclerosis; ESI: Exit-site infection; ESRD: End-stage renal disease; F: Female; GM: Gentamicin; HD: Hemodialysis; IPM/CS: Imipenem/cilastatin; LVFX: Levofloxacin; M: Male; M. abscessus: Mycobacterium abscessus; MEPM: Meropenem;

MFLX: Moxifloxacin; MRSA: Methicillin-resistant Staphylococcus aureus; N/ A: Not applicable; NTM: Nontuberculous mycobacteria; PD: Peritoneal dialysis; Ref: References; SBT/ABPC: Sulbactam/ampicillin; TA: Tunnel abscess; TAZ/

PIPC: Tazobactam/piperacillin; TEIC: Teicoplanin; TGC: Tigecycline; TGF$\beta$ : Transforming growth factor- $\beta$; VCM: Vancomycin; VEGF: Vascular endothelial growth factor

\section{Acknowledgements}

The authors are grateful to Dr. Keiji Mimura (Kaizuka-Nishide Clinic, Kaizuka, Japan) for his kind support in the treatment of the case 1 patient and A. Yamamoto, S. Ogino, and Y. Mizukami in the Department of Nephrology, Kyoto University Graduate School of Medicine, for their secretarial assistance.

Funding

This work was supported in part by EXT/JSPS KAKENHI Grant Number 17 K16079 for writing the manuscript.

Availability of data and materials

All of the data and materials are included in the manuscript.

Authors' contributions

EO, EU, and KPM wrote the manuscript. HY, NT, KK, MK, TM, and MY contributed to the treatment of patients and discussion and reviewed the manuscript. All authors read and approved the final version of the manuscript.

Ethics approval and consent to participate

The case report was written in compliance with the Declaration of Helsinki.

Consent for publication

Consent for publication of this case report was obtained from the patients.

Competing interests

The authors declare that they have no competing interests.

\section{Publisher's Note}

Springer Nature remains neutral with regard to jurisdictional claims in published maps and institutional affiliations.

\section{Author details}

'Department of Nephrology, Graduate School of Medicine, Kyoto University, 54 Shogoin Kawahara-cho, Sakyo-ku, Kyoto 606-8507, Japan. ${ }^{2}$ Medical Innovation Center, Graduate School of Medicine, Kyoto University, 53 Shogoin Kawahara-cho, Sakyo-ku, Kyoto 606-8507, Japan. ${ }^{3}$ Department of Nephrology, Osaka Red Cross Hospital, 5-30 Hudegasaki-cho, Tennoji-ku, Osaka 543-8555, Japan. ${ }^{4}$ Institute for Clinical and Translational Science, Nara Medical University, 840 Shijo-Cho, Kashihara, Nara 634-8521, Japan.

Received: 28 June 2018 Accepted: 19 November 2018

Published online: 10 December 2018

References

1. Li PK, Szeto CC, Piraino B, de Arteaga J, Fan S, Figueiredo AE, et al. ISPD peritonitis recommendations: 2016 update on prevention and treatment. Perit Dial Int. 2016;36:481-508. 
2. Brown-Elliott BA, Wallace RJ Jr. Clinical and taxonomic status of pathogenic nonpigmented or late-pigmenting rapidly growing mycobacteria. Clin Microbiol Rev. 2002;15:716-46.

3. Wallace RJ Jr, Swenson JM, Silcox VA, Good RC, Tschen JA, Stone MS. Spectrum of disease due to rapidly growing mycobacteria. Rev Infect Dis. 1983:5:657-79.

4. Lo MW, Mak SK, Wong YY, Lo KC, Chan SF, Tong GM, et al. Atypical mycobacterial exit site infection and peritonitis in peritoneal dialysis patients on prophylactic exit site gentamicin cream. Perit Dial Int. 2013;33:267-72.

5. Yang TK, Lee JJ, Lu PL, Kuo HT, Kuo MC, Chen HC. Peritoneal dialysisassociated peritonitis caused by Mycobacterium abscessus. Perit Dial Int. 2015;35:369-71.

6. Renaud CJ, Subramanian S, Tambyah PA, Lee EJ. The clinical course of rapidly growing nontuberculous mycobacterial peritoneal dialysis infections in Asians: a case series and literature review. Nephrology. 2011;16:174-9.

7. Kameyama H, Mori Y, Kimura T, Sugishita C, Adachi T, Sonomura K, et al. A case report of Mycobacterium abscessus peritonitis in a peritoneal dialysis patient. Ther Apher Dial. 2007;11:449-51.

8. Siddiqi N, Sheikh I. Peritonitis caused by Mycobacterium abscessus in patients on continuous ambulatory peritoneal dialysis. Saudi J Kidney Dis Transplant. 2012;23:321-4.

9. Jiang SH, Roberts DM, Clayton PA, Jardine M. Non-tuberculous mycobacterial PD peritonitis in Australia. Int Urol Nephrol. 2013;45:1423-8.

10. Mooren VHJF, Bleeker MWP, van Ingen J, Hermans MHA, Wever PC Disseminated Mycobacterium abscessus infection in a peritoneal dialysis patient. IDCases. 2017:9:6-7.

11. Slagle KM, Oblack DL. Mycobacterium abscessus peritonitis: a case report. Clin Lab Sci. 1998;11:206-8.

12. Vera G, Lew SQ. Mycobacterium fortuitum peritonitis in two patients receiving continuous ambulatory peritoneal dialysis. Am J Nephrol. 1999;19:586-9.

13. Bryant JM, Grogono DM, Rodriguez-Rincon D, Everall I, Brown KP, Moreno P, et al. Emergence and spread of a human-transmissible multidrug-resistant nontuberculous mycobacterium. Science. 2016;354:751-7.

14. Johnson MM, Odell JA. Nontuberculous mycobacterial pulmonary infections. J Thorac Dis. 2014;6:210-20.

15. Griffith DE, Aksamit T, Brown-Elliott BA, Catanzaro A, Daley C, Gordin F, et al.; ATS Mycobacterial Diseases Subcommittee; American Thoracic Society; Infectious Disease Society of America. An official ATS/IDSA statement: diagnosis, treatment, and prevention of nontuberculous mycobacterial diseases. Am J Respir Crit Care Med 2007;175:367-416.

16. Woo PC, Leung KW, Wong SS, Chong KT, Cheung EY, Yuen KY. Relatively alcohol-resistant mycobacteria are emerging pathogens in patients receiving acupuncture treatment. J Clin Microbiol. 2002;40:1219-24.

17. Cheng A, Sun HY, Tsai YT, Wu UI, Chuang YC, Wang JT, et al. In vitro evaluation of povidone-iodine and chlorhexidine against outbreak and nonoutbreak strains of Mycobacterium abscessus using standard quantitative suspension and carrier testing. Antimicrob Agents Chemother. 2017;62:e01364-17.

18. Io H, Hamada C, Ro Y, Ito Y, Hirahara I, Tomino Y. Morphologic changes of peritoneum and expression of VEGF in encapsulated peritoneal sclerosis rat models. Kidney Int. 2004:65:1927-36.

19. Toda N, Mori K, Kasahara M, Koga K, Ishii A, Mori KP, et al. Deletion of connective tissue growth factor ameliorates peritoneal fibrosis by inhibiting angiogenesis and inflammation. Nephrol Dial Transplant. 2018;33:943-53.

20. Lin CH, Shu CC, Hsu CL, Cheng SL, Wang JY, Yu CJ, Lee LN. The trend and the disease prediction of vascular endothelial growth factor and placenta growth factor in nontuberculous mycobacterial lung disease. Sci Rep. 2016;6:37266.

21. Bar-On O, Mussaffi H, Mei-Zahav M, Prais D, Steuer G, Stafler P, et al. Increasing nontuberculous mycobacteria infection in cystic fibrosis. J Cyst Fibros. 2015;14:53-62.

Ready to submit your research? Choose BMC and benefit from:
- fast, convenient online submission
- thorough peer review by experienced researchers in your field
- rapid publication on acceptance
- support for research data, including large and complex data types
- gold Open Access which fosters wider collaboration and increased citations
- maximum visibility for your research: over 100M website views per year
At BMC, research is always in progress.
Learn more biomedcentral.com/submissions

